Pacific Journal of Mathematics

RANDOM POINTS IN A SIMPLEX 


\title{
RANDOM POINTS IN A SIMPLEX
}

\author{
W. J. REED
}

The expected values of certain functions of $N$ points chosen at random in an $n$ dimensional simplex or parallelotope are considered, and a decomposition of such integrals is obtained by use of a generalised form of Crofton's Theorem.

Explicit expressions are found for the moments of the area of the triangle formed by three points chosen at random in a triangle or parallelogram.

I. Introduction. In Euclidean $n$-space a convex body $K_{n}$ of volume $V$ is given, and $n+1$ points

$$
\mathbf{x}_{1}, \mathbf{x}_{2}, \ldots \ldots . ., \mathbf{x}_{n+1}
$$

are chosen independently, at random in $K_{n}$.

Let $C\left(\mathbf{x}_{1}, \mathbf{x}_{2}, \ldots, \mathbf{x}_{n+1}\right)$ denote the volume of the convex hull of the $n+1$ points, which with probability one is an $n$-simplex, and let

$$
D\left(K_{n}\right)=C\left(\mathbf{x}_{1}, \mathbf{x}_{2}, \ldots, \mathbf{x}_{n+1}\right) / V .
$$

Let $V_{K_{n}}^{h}$ be the $h$ th moment of $D\left(K_{n}\right)$

$$
V_{K_{n}}^{h}=E\left(\left[D\left(K_{n}\right)\right]^{h}\right)
$$

Since a ratio of volumes, and thus a uniform distribution over a body, is preserved under affine transformation, it follows that $V_{K_{n}}^{h}$ is an affine invariant of $K n$.

The problem of finding $V_{K_{n}}^{h}$ is almost trivial for the case $n=1$, for $K_{1}$ is a line segment.

$$
V_{K_{1}}^{h}=\frac{2}{(h+1)(h+2)} \text {. }
$$

For the case of $n=2$, the problem of finding the first moment $V_{K_{2}}^{1}$, for various plane convex figures $K_{2}$, was investigated by Sylvester and others during the 1860's and has come to be known as Sylvester's Problem (see[3] for references).

For the equivalence class of triangles $\Delta_{2}$ it is known that 


$$
V_{\Delta_{2}}^{1}=\frac{1}{12}
$$

and for the ellipse $B_{2}$

$$
V_{B_{2}}^{1}=\frac{35}{48} \pi^{2}
$$

In higher dimensions the problem is more difficult. Klee [4] considers the problem, and in particular asks for $V_{\Delta_{n}}^{1}$ where $\Delta_{n}$ is the $n$-simplex.

Kingman [5] shows that for the $n$-dimensional ellipsoid $B_{n}$

$$
V_{B_{n}}=\left(\begin{array}{c}
n+1 \\
\frac{1}{2}(n+1)
\end{array}\right)^{n+1} /\left(\begin{array}{c}
(n+1)^{2} \\
\frac{1}{2}(n+1)^{2}
\end{array}\right) 2^{n}
$$

He also shows that the second moment $V_{K_{n}}^{2}=(n+1) I / n ! V^{n+2}$, where $I$ is the determinant of the inertia matrix of $K_{n}$ regarded as a body of uniform density.

One way of tackling Sylvester's problem in two dimensions has been with the use of Crofton's Theorem (see [1] and [3]). This sets up a differential equation involving the expected value of $D\left(K_{2}\right)$ when one of the points, $x_{1}$ say, is chosen according to some probability law on the boundary of $K_{2}$, and $\mathrm{x}_{2}$ and $\mathrm{x}_{3}$ are chosen independently, at random from the interior of $K_{2}$. This reduces the total number of degrees of freedom of the random points by one, i.e. it reduces the number of integrations involved by one.

In this paper it is shown how a generalized form of Crofton's Theorem can be repeatedly applied, to simplify the problem of finding $V_{K_{n}}^{h}$ when $K_{n}$ is the $n$-simplex $\Delta_{n}$ or the $n$ dimensional parallelotope $P_{n}$.

In particular, in two dimensions it is shown for the triangle $\Delta_{2}$, how $V_{\Delta_{2}}^{h}$ can be expressed as a linear combination of two basic expectations, in each of which the number of degrees of freedom of the random points is reduced by three. These basic expectations are found, and it is shown that

$$
V_{\Delta_{2}}^{h}=\frac{12}{(h+1)^{3}(h+2)^{3}(h+3)(2 h+5)}\left\{6(h+1)^{2}+(h+2)^{2} \sum_{r=0}^{h} \frac{1}{\left(\begin{array}{l}
h \\
r
\end{array}\right)^{2}}\right\}
$$

A similar decomposition into two basic expectations holds for the parallelogram $P_{2}$, and it is shown that

$$
V_{P_{2}}^{h}=\frac{3}{(h+1)(h+2)^{3}(h+3)^{2} 2^{h-2}}\left\{(h+1)+2(h+2) \sum_{r=1}^{h+1} \frac{1}{r}\right\}
$$


It is shown how in principle, a decomposition of $V_{K_{n}}^{h}$ into a linear combination of basic expectations in each of which the number of degrees of freedom is reduced by $n+1$, could be obtained when $K_{n}$ is either the $n$-simplex $\Delta_{n}$ or the $n$-dimensional parallelotope $P_{n}$.

However for $n>2$ the evaluation of these basic expectations seems difficult.

In II an analytic formulation of the problem for the $n$-simplex $\Delta_{n}$ is developed along with a suitable notation.

In III the generalized Crofton theorem is used to obtain expressions for $V_{K_{2}}^{h}$ for the triangle and the parallelogram.

In IV the problem in higher dimensions is considered, and some numerical results given.

II. Random points in the $n$-simplex $\Delta_{r}$ The ratio of the volumes of two bodies in $n$-space is invariant under a nonsingular affine transformation. It follows that a uniform distribution over a body will be preserved under such a transformation.

Since any nondegenerate $n$-simplex $\Delta_{n}$ is affinely equivalent to a regular $n$-simplex $S_{n}$ it follows that

$$
D\left(\Delta_{n}\right)=C\left(\mathbf{y}_{1}, \ldots, \mathbf{y}_{n+1}\right) /\left(\text { volume of } S_{n}\right)
$$

where $\mathbf{y}_{1}, \mathbf{y}_{2} \ldots, \mathbf{y}_{n+1}$ are $n+1$ points chosen independently at random in $S_{n}$.

Let $X_{1}, X_{2}, \ldots, X_{k}$ be $k$ independent random variables with distribution function

$$
F(x)= \begin{cases}0 & , x<0 \\ 1-e^{-x}, & x \geq 0\end{cases}
$$

and let

$$
Y_{j}=\frac{X_{j}}{\sum_{i=1}^{k} X_{i}}
$$$$
(j=1, \ldots, k)
$$

The $k$-dimensional vector random variable

$$
\mathbf{Y}=\left(\begin{array}{c}
Y_{1} \\
\vdots \\
Y_{k}
\end{array}\right)
$$

has a uniform distribution over the regular $(k-1)$-simplex 


$$
S_{k-1}=\left\{\left(\begin{array}{c}
x_{1} \\
\vdots \\
x_{k}
\end{array}\right): x_{j} \geq 0 \quad(j=1, \ldots, k), \sum_{j=1}^{k} x_{j}=1\right\}
$$

and furthermore $\mathbf{Y}$ is independent of the denominator

$$
\sum_{i=1}^{k} X_{i}
$$
[10]).)

(This is a particular case of a $k$-variate Dirichlet distribution (see

Suppose $\mathbf{y}_{1}, \mathbf{y}_{2}, \ldots, \mathbf{y}_{n+1}$ are the Cartesian column vectors of $n+1$ points in $S_{n}$. It can be shown that the ratio of the volume of the convex hull of these $n+1$ points to the volume of $S_{n}$ is

$$
\left|\operatorname{det}\left(\mathbf{y}_{1}, \mathbf{y}_{2}, \ldots \mathbf{y}_{n+1}\right)\right|
$$

and hence $D\left(\Delta_{n}\right)$ can be expressed

$$
D\left(\Delta_{n}\right)=\frac{1}{\prod_{j=1}^{n+1}\left(\sum_{i=1}^{n+1} X_{i j}\right)}\left|\operatorname{det}\left(\left(X_{i j}\right)\right)_{i, j=1, \ldots, n+1}\right|
$$

where $X_{i j}(i, j=1, \ldots, n+1)$ are $(n+1)^{2}$ independent random variables with distribution function $F(x)$, and furthermore it can be shown that $D\left(\Delta_{n}\right)$ is independent of its denominator

$$
\prod_{j=1}^{n+1}\left(\sum_{i=1}^{n+1} X_{i j}\right)
$$

Since the sum of independent exponential random variables has a gamma distribution it follows that the $h$ th moment of $D\left(\Delta_{n}\right)$, will be

$$
V_{\Delta_{n}}^{h}=\frac{E\left\{\left|\operatorname{det}\left(\left(X_{i j}\right)\right)_{i, j=1, \ldots, n+1}\right| h \mid\right.}{[(n+h) ! / n !]^{n+1}} .
$$

The second moment of the determinant of a matrix of independent, identically distributed random variables is known and thus

$$
V_{\Delta_{n}}^{2}=\frac{n !}{(n+1)^{n}(n+2)^{n}}
$$


Higher even order moments $V_{\Delta_{n}}^{2 h}$ may admit a solution, by similar methods, but odd order moments look very difficult due to the presence of the absolute value sign.

However, as pointed out by Kingman [5] the even order moments determine the distribution of $D\left(\Delta_{n}\right)$ and thus determine the odd order moments in principle, although this hardly seems to offer a practical method of solving Klee's problem of finding $V_{\Delta_{n}}^{1}$.

It is obvious that if

$$
X_{1}=0
$$

and $X_{j}(j=2, \ldots, n+1)$ are independent r.vs with df. $F(x)$, then the $(n+$ 1)-dimensional vector r.v.

$$
\mathbf{Y}=\left(\begin{array}{c}
Y_{1} \\
\vdots \\
Y_{n+1}
\end{array}\right)
$$

where

$$
Y_{j}=\frac{X_{j}}{\sum_{i=1}^{n+1} X_{i}}, \quad(j=1, \ldots, n)
$$

represents a point at random in the $(n-1)$ simplex $S_{n-1}$ which forms the "face" of $S_{n}$ opposite to the vertex

$$
\left(\begin{array}{c}
1 \\
0 \\
\vdots \\
0
\end{array}\right)
$$

Similarly if $X_{1}=X_{2}=0$, all other $X_{j}$ independent r.vs. with df. $F(x)$, then $Y$ represents a point at random in the $(n-2)$-simplex $S_{n-2}$ which forms that part of the boundary of $S_{n}$ opposite to the "edge" joining vertices

$$
\left(\begin{array}{c}
1 \\
0 \\
\vdots \\
0
\end{array}\right) \text { and }\left(\begin{array}{c}
0 \\
1 \\
0 \\
\vdots \\
0
\end{array}\right) \text {. }
$$

Let $X_{1, i_{1}}=X_{2, i_{2}}=\ldots=X_{k, i_{k}}=0, k \leq n+1$ for some sequence $\left(i_{1}, i_{2}, \ldots, i_{k}\right)$ and suppose all other $X_{i j}$ are r.vs with d.f. $F(x)$. 
Define the vectors

$$
\mathbf{Y}_{j}=\left(\begin{array}{c}
Y_{1, j} \\
\vdots \\
Y_{n+1, j}
\end{array}\right) \quad \text { for } j=1, \ldots, n+1
$$

where

$$
Y_{i j}=\frac{X_{i j}}{\sum_{i=1}^{n+1} X_{i j}}
$$

and define the matrix $M_{n}\left(i_{1}, \ldots, i_{k}\right)=\left(Y_{1}, \ldots, Y_{n+1}\right)$.

This matrix represents $n+1$ random points each in some simplex of the boundary $S_{n}$ or in $S_{n}$ itself. Note that no two points can have a common zero barycentric coordinate, since $M_{n}$ has at most one zero in each row.

We shall be concerned only with functions of $n+1$ points in $S_{n}$ which:

(a) are symmetric in the sense of being invariant under permutations of the points.

(b) depend only on intrinsic properties of the points and their relative positions and not on their positions relative to $S_{n}$.

Such a function will be invariant under permutations of rows and columns of the configuration matrix $M_{n}\left(i_{1}, \ldots, i_{k}\right)$.

It should be fairly clear that by permuting rows and columns of the matrix $M_{n}$ it can be arranged that the sequence $\left(i_{1}, \ldots, i_{k}\right)$ satisfies the following.

(1) it is nondecreasing.

(2) for each $r, 1 \leq r \leq i_{k}$, there exists $j, 1 \leq j \leq k$, such that $i_{j}=r$.

(3) for each $r, 1 \leq r \leq i_{k}$ define $m(r)$ as the number of $i_{j}$ 's which equal $r$. Then for each $r, s$ such that $1 \leq r<s \leq i_{k}$ it follows that $m(r) \geq m(s)$.

Under the operations of permuting points or relabelling vertices, any sequence of $k$ positive integers, $k \leq n+1$, satisfying (1), (2) and (3) above will determine an equivalence class in the set of configurations of $n+1$ random points chosen either in $S_{n}$ or in a simplex of the boundary of $S_{n}$ in such a way that no two points have a common zero barycentric coordinate.

For a function $\phi$ satisfying (a) and (b) and any sequence $\left(i_{1}, \ldots, i_{k}\right)$ satisfying (1), (2) and (3) we define

$$
\mu_{n}\left(i_{1}, \ldots, i_{k}\right)=E\left\{\phi\left(M_{n}^{*}\left(i_{1}, \ldots, i_{k}\right)\right\}\right.
$$

where $M_{n}^{*}\left(i_{1}, \ldots, i_{k}\right)$ is any matrix obtained by permuting rows and columns of $M_{n}\left(i_{1}, \ldots, i_{k}\right)$.

We also define $\mu_{n}=E\left\{\phi\left(\mathbf{Y}_{b}, \ldots, \mathbf{Y}_{n+1}\right)\right\}$ if all $X_{i j}$ have d.f. $F(x), i, j=$ $1, \ldots, n+1$. 
To illustrate the notation we show the case of $n=2$. The dots in the matrix represent the nonzero $Y_{i, j}$.

\begin{tabular}{clll}
$\begin{array}{c}\text { Expected Value } \\
\text { of } \phi\end{array}$ & $\begin{array}{l}\text { Configuration } \\
\text { Matrix }\end{array}$ & $S_{2}$ & Situation \\
\hline
\end{tabular}

$\mu_{2}$
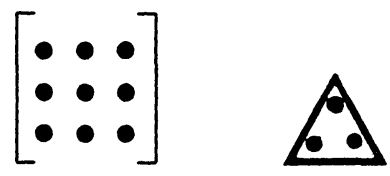

3 points at random in $S_{2}$.

$\mu_{2}(1)$

$$
M_{2}(1)=\left[\begin{array}{lll}
0 & \bullet & \bullet \\
\bullet & \bullet & \bullet \\
\bullet & \bullet & \bullet
\end{array}\right]
$$

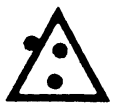

2 points at random in $S_{2}$, one at random on an edge.

$\mu_{2}(1,1) \quad M_{2}(1,1)=\left[\begin{array}{lll}0 & \bullet & \bullet \\ 0 & \bullet & \bullet \\ \bullet & \bullet & \bullet\end{array}\right]$

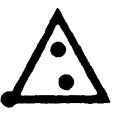

2 points at random in $S_{2}$, one fixed at a vertex.

$\mu_{2}(1,2)$

$$
M_{2}(1,2)=\left[\begin{array}{lll}
0 & \bullet & \bullet \\
\bullet & 0 & \bullet \\
\bullet & \bullet & \bullet
\end{array}\right]
$$

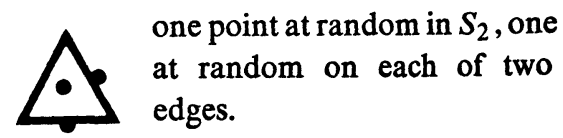

$\mu_{2}(1,1,2) \quad M_{2}(1,1,2)=\left[\begin{array}{lll}0 & \bullet & \bullet \\ 0 & \bullet & \bullet \\ \bullet & 0 & \bullet\end{array}\right]$

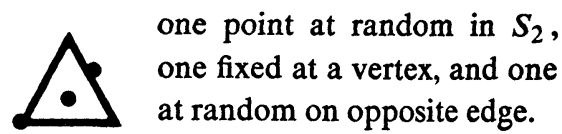

$\mu_{2}(1,2,3) \quad M_{2}(1,2,3)=\left[\begin{array}{lll}0 & \bullet & \bullet \\ \bullet & 0 & \bullet \\ \bullet & \bullet & 0\end{array}\right]$

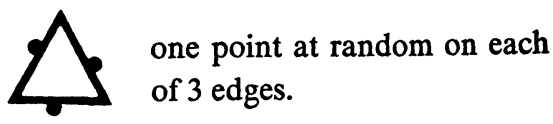

It will be shown how $\mu_{n}$ can be decomposed into a linear combination of basic expectations

$$
\mu_{n}\left(i_{1}, \ldots, i_{n+1}\right)
$$

III. Application of a generalized form of Crofton's theorem. Crofton's Theorem (see [1], [3]) gives a differential relationship between the expected value of a function of $N$ points chosen independently at random in a domain $D$, and the expected value of that function when one of the points is constrained to the boundary of $D$ and the other $N-1$ are selected at random in $D$.

In [6] the following more general theorem is proved. 
Theorem. Suppose that for $j=1, \ldots, k, D_{j}$ is a domain in $n_{j}$-dimensional Euclidean space, with volume $V_{j}$, and that $N_{j}$ points are chosen independently at random in $D_{j}$.

Let $\mu$ be the expected value of some numerical function of the $\Sigma_{j=1}^{k} N_{j}$ points, which depends only on intrinsic properties of the points and their relative positions and not on the domains $D_{j}$ or the positions of the points relative to them, and suppose that under an affine transformation $\mu$ changes only through $V_{1}, \ldots, V_{k}$.

If each domain $D_{j}$ is incremented by an infinitesimal around $\Delta V_{j}, j=$ $1, \ldots, k$, in such $a$ way as to preserve affine equivalence with the original situation, then

$$
\Delta \mu=\sum_{j=1}^{k} N_{j}\left(\mu_{j}^{*}-\mu\right) \frac{\Delta V_{j}}{V_{j}}
$$

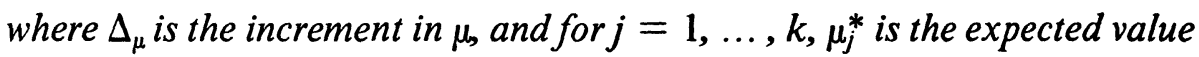
of the function when one point is chosen at random in the increment of $D_{j}$, $N_{j}-1$ points are chosen at random in $D_{j}$, and $N_{l}$ points are chosen at random in $D_{l}$, for $l \neq j, l=1, \ldots, k$.

The theorem is now applied in finding the expected value of functions of three random points in the triangle $\Delta_{2}$.

Let $V$ be the area of a regular (equilateral) triangle $S_{2}$, and let $s$ be the length of the sides. Let $\phi$ be a symmetric function of three points in $S_{2}$ depending only on intrinsic properties of the points, and let $\mu_{2}$ be the expected value of $\phi$, which we shall assume changes under an affine transformation only through $V$.

We apply the theorem with three points in one domain $\left(S_{2}\right)$, incrementing $S_{2}$ by a narrow band of area $\Delta V$ parallel to one of the edges. (This is simply Crofton's original theorem.)

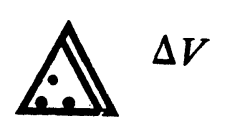

Using the notation developed in II, we have

$$
\Delta \mu_{2}=3\left(\mu_{2}(1)-\mu_{2}\right) \frac{\Delta V}{V}
$$

from which we obtain

$$
\frac{d}{d V}\left\{V^{3} \mu_{2}\right\}=3 \mu_{2}(1) V^{2} .
$$


We now apply the theorem again for $\mu_{2}(1)$. The situation is now that there are two points at random in $S_{2}$, and one point at random in the side of $S_{2}$. Incrementing $S_{2}$ along an edge other than the one containing a point we obtain

$$
\Delta \mu_{2}(1)=2\left[\mu_{2}(1,2)-\mu_{2}(1)\right] \frac{\Delta V}{V}+1 \cdot\left[\mu_{2}(1,1)-\mu_{2}(1)\right] \frac{\Delta s}{s}
$$

and since $V=s^{2} \sqrt{3} / 4$ it follows that $\Delta V / V=2 \Delta s / s$ and hence

$$
\frac{d}{d V}\left\{V^{5 / 2} \mu_{2}(1)\right\}=\left[2 \mu_{2}(1,2)+\frac{1}{2} \mu_{2}(1,1)\right] V^{3 / 2} \text {. }
$$

Applying the theorem again for $\mu_{2}(1,2)$ incrementing $S_{2}$ along the edge containing no point gives

$$
\begin{gathered}
\Delta \mu_{2}(1,2)=\left[\mu_{2}(1,2,3)-\mu_{2}(1,2)\right] \frac{\Delta V}{V}+\left[\mu_{2}(1,1,2)-\mu_{2}(1,2)\right] \frac{\Delta s}{s} \\
+\left[\mu_{2}(1,1,2)-\mu_{2}(1,2)\right] \frac{\Delta s}{s}
\end{gathered}
$$

which gives

$$
\frac{d}{d V}\left\{V^{2} \mu_{2}(1,2)\right\}=\left[\mu_{2}(1,2,3)-\mu_{2}(1,1,2)\right] V .
$$

We apply the theorem again for $\mu_{2}(1,1)$ incrementing $S_{2}$ along the edge opposite to the vertex at which the point is fixed.

This gives

which gives

$$
\Delta \mu_{2}(1,1)=2\left[\mu_{2}(1,1,2)-\mu_{2}(1,1)\right] \frac{\Delta V}{V}
$$

$$
\frac{d}{d V}\left\{V^{2} \mu_{2}(1,1)\right\}=2 \mu_{2}(1,1,2) V
$$

If the expectations $\mu_{2}(1,2,3)$ and $\mu_{2}(1,1,2)$ can be determined for a given function of the random points, then in principle it should be possible to determine $\mu_{2}$ by integrating equations (1) through (4).

Since a distribution function can be expressed as the expected value of an indicator random variable, in principle we can determine the distribution function of a given function of the random points if we can determine it in the two constrained cases. 
Before considering these constrained expectations we make some remarks concerning the way in which the theorem has been applied.

Firstly we have always incremented $S_{2}$ along an edge to which no points are constrained. A close examination of the proof of the theorem [6] will reveal that it would not be valid to apply the theorem if this were not the case. This is because the expectation in the case with the enlarged domain must decompose, to first order, into two parts-one is $\mu^{*}$ the constrained expectation and the other reduces to $\mu$, the expectation with the original domains. If we were to increment $S_{2}$ along an edge containing a point the latter part would be fundamentally different from $\mu$.

Secondly we have always incremented $S_{2}$ parallel to an edge. This ensures that the enlarged domain is still a regular triangle and that in the resulting constrained case the point will be chosen at random from the edge. However the regularity of the enlarged domain is not essential, since we assume that within an affine equivalence class $\mu$ depends only on $V$. Thus it would be sufficient that the enlarged domain be any triangle. This would be the case if $S_{2}$ were enlarged by a wedge-shaped increment.

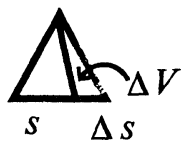

In this case the resulting constrained point will have a distribution with a linear density function along the edge, and also $\Delta V / V=\Delta s / s$. In this way it is possible to decompose $\mu_{2}(1,1,2)$ further, but not $\mu_{2}(1,2,3)$ because of our earlier remark about the validity of the theorem's application.

We now solve (1) to (4) for the case when $\phi$ is a function which varies with $V$ proportionally to $V^{h}$.

In this case $\mu_{2}=\hat{\mu}_{2} V^{h}$

$$
\mu_{2}(1)=\hat{\mu}_{2}(1) V^{h}
$$

etc., where the $\hat{\mu}_{2}$ 's are independent of $V$. We can rewrite (1) to (4) as

$$
\begin{aligned}
\frac{d}{d V}\left\{\hat{\mu}_{2} V^{h+3}\right\} & =3 \hat{\mu}_{2} V^{h+2} \\
\frac{d}{d V}\left\{\hat{\mu}_{2}(1) V^{5 / 2+h}\right\} & =\left[2 \hat{\mu}_{2}(1,2)+\frac{1}{2} \hat{\mu}_{2}(1,1)\right] V^{h+3 / 2} \\
\frac{d}{d V}\left\{\hat{\mu}_{2}(1,2) V^{h+2}\right\} & =\left[2 \hat{\mu}_{2}(1,2,3)+\hat{\mu}_{2}(1,1,2)\right] V^{h+1}
\end{aligned}
$$




$$
\frac{d}{d V}\left\{\hat{\mu}_{2}(1,1) V^{h+2}\right\}=2 \hat{\mu}_{2}(1,1,2) V^{h+1} .
$$

Solving these and eliminating $\hat{\mu}_{2}(1), \hat{\mu}_{2}(1,2)$ and $\hat{\mu}_{2}(1,1)$ gives

$$
\hat{\mu}_{2}=\frac{6}{(h+2)(h+3)(2 h+5)}\left[3 \hat{\mu}_{2}(1,1,2)+2 \hat{\mu}_{2}(1,2,3)\right]
$$

and a similar equation holds for the $\mu_{2}, \mu_{2}(1,1,2)$ and $\mu_{2}(1,2,3)$.

It seems that this is not altogether a new result. In a footnote [2] Crofton mentions that J. J. Sylvester has discovered "remarkable results" which he calls a "decomposition of probabilities." This is essentially (5) with $h=0$, the $\mu_{2}$ 's being in this case probabilities, which can be thought of as expectations of indicator functions, independent of $V$.

There is a vague general account of a paper Sylvester read to the British Association [7] concerning the result. He used the result in a solution to a problem in the Educational Times, to find $V_{\Delta_{2}}^{1}[8]$ and proposes a question in a later issue of that journal [9] which asks for a proof. No proof was forthcoming and as far as can be discovered Sylvester never gave any further details.

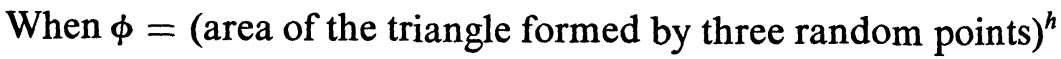

$$
V_{\Delta_{2}}^{h}=E\left(\frac{\varphi}{V^{h}}\right)=\hat{\mu}_{2} .
$$

The basic expectations $\hat{\mu}_{2}(1,1,2)$ and $\hat{\mu}_{2}(1,2,3)$ are easily found in this case. By the methods of II

$$
\begin{gathered}
\hat{\mu}_{2}(1,1,2)=\frac{E\left\{\left|\operatorname{det}\left[\begin{array}{lll}
0 & X_{12} & X_{13} \\
0 & X_{22} & X_{23} \\
X_{31} & 0 & X_{33}
\end{array}\right]\right|^{h}\right\}}{E\left\{X_{31}^{h}\right\} E\left\{\left(X_{12}+X_{22}\right)^{h}\right\} E\left\{\left(X_{13}+X_{23}+X_{33}\right)^{h}\right\}} \\
=\frac{V_{K_{1}}^{h} E\left\{\left(X_{13}+X_{23}\right)^{h}\right\}}{E\left\{\left(X_{13}+X_{23}+X_{33}\right)^{h}\right\}}
\end{gathered}
$$

since

$$
V_{K_{1}}^{h}=E\left\{\left|\operatorname{det}\left[\begin{array}{ll}
X_{12} & X_{13} \\
X_{22} & X_{23}
\end{array}\right]\right|^{h}\right\} \mid E\left\{\left(X_{12}+X_{22}\right)^{h}\right\} E\left\{\left(X_{13}+X_{23}\right)^{h}\right\} .
$$


Thus

$$
\hat{\mu}(1,1,2)=\frac{2}{(h+1)(h+2)} \frac{\Gamma(h+2)}{\Gamma(2)} \frac{\Gamma(3)}{\Gamma(h+3)}=\frac{4}{(h+1)(h+2)^{2}}
$$

Also

$$
\begin{aligned}
\hat{\mu}_{2}(1,2,3) & =\frac{E\left\{\left.\left|\operatorname{det}\left[\begin{array}{lll}
0 & X_{12} & X_{13} \\
X_{21} & 0 & X_{23} \\
X_{31} & X_{32} & 0
\end{array}\right]\right|\right|^{h}\right\}}{\left.E\left\{\left(X_{21}+X_{31}\right)^{h}\right\} E\left\{\left(X_{12}+X_{32}\right)^{h}\right\} E\left(X_{13}+X_{23}\right)^{h}\right\}} \\
& =E\left\{\left(X_{12} X_{23} X_{31}+X_{13} X_{21} X_{32}\right)^{h}\right\} /[(h+1) !]^{3} \\
& =\frac{1}{[(h+1) !]^{3}} \sum_{r=0}^{h}\left(\begin{array}{l}
h \\
r
\end{array}\right) E\left\{X_{12}^{r} X_{23}^{r} X_{31}^{r} X_{13}^{h-r} X_{21}^{h-r} X_{32}^{h-r}\right\} \\
& =\frac{1}{(h+1)^{3}} \sum_{r=0}^{h} \frac{1}{\left(\begin{array}{l}
h \\
r
\end{array}\right)^{2}} .
\end{aligned}
$$

From (6), (7), and (8),

$$
V_{\Delta_{2}}^{h}=\frac{12}{(h+1)^{3}(h+2)^{3}(h+3)(2 h+5)}\left\{6(h+1)^{2}+(h+2)^{2} \sum_{r=0}^{h} \frac{1}{\left(\begin{array}{l}
h \\
r
\end{array}\right)^{2}}\right\} .
$$

A similar decomposition can be obtained for the affine equivalence class of parallelograms, $P_{2}$. Repeated application of the theorem for functions proportional to $V^{h}$ gives

$$
\mu=\frac{12}{(h+2)^{2}(h+3)^{2}}\left(\mu^{*}+2 \mu^{* *}\right)
$$

where $\mu^{*}$ is the expectation when a point is fixed at each of two opposite vertices, and a third point is chosen at random from the interior, and $\mu^{* *}$ is the expectation when one point is fixed at a vertex and a point is chosen at random from each of the two sides non-incident with that vertex.

The $h$ th moment of the area of the triangle formed by the three points in each of the above situations is easily found by considering the configurations in the square. From this we obtain 


$$
V_{P_{2}}^{h}=\frac{3}{(h+1)(h+2)^{3}(h+3)^{2} 2^{h-2}}\left\{(h+1)+2(h+2) \sum_{r=1}^{h+1} \frac{1}{r}\right\} \text {. }
$$

IV. Application of the theorem in higher dimensions. In higher dimensions the problem does not admit such a simple solution.

Consider the general case of the simplex $S_{n}$ in $n$ dimensions. The generalized Crofton theorem can be repeatedly applied. Bearing in mind the remarks in III about the validity of the application of the theorem we see that $S_{n}$ can be incremented parallel to a "face" named by the vertex opposite to it only if none of the $n+1$ points has zero barycentric coordinate for that vertex. Thus at each iteration of the theorem a new expectation arises for a situation with a configuration matrix (as described in II) with one extra zero in a row which did not previously contain one.

When each row of the configuration matrix contains exactly one zero we will have exhausted the possibilities obtainable by incrementing $S_{n}$ parallel to one of its faces. We will call these the basic expectations. Of course it will be possible to decompose some of these basic expectations further by incrementing $S_{n}$ in other ways, but these cases seem rather difficult to deal with.

If we are considering only intrinsic, symmetric functions of the $n+1$ points it will be seen that each basic expectation can be represented

$$
\mu_{n}\left(i_{1}, i_{2}, \ldots, i_{n+1}\right)
$$

where $i_{j}, j=1, \ldots, n+1$ satisfy the properties (1), (2), (3) of II, with the further restriction that they do not all equal one. And furthermore it will be seen that each such sequence represents a basic expectation which can be arrived at by successive decomposition, using the theorem.

Thus the number of basic expectations will be the number of such sequences

viz

$$
p(n+1)-1
$$

where $p(m)$ is the number of ways the integer $m$ can be partitioned into integral parts, the order of the parts being immaterial.

For example, 3 is capable of partition as $(1,1,1),(1,2)$, (3) leading to the basic expectations $\mu_{2}(1,2,3)$ and $\mu_{2}(1,1,2)$ for the case $n=2$. (The last partition (3) is discarded since it yields 3 zeros in one column.)

Again 4 is capable of partition as $(1,1,1,1),(1,1,2),(1,3),(2,2),(4)$, and in the case $n=3$ we have the following corresponding basic expectations 


$$
\mu_{3}(1,2,3,4), \mu_{3}(1,1,2,3), \mu_{3}(1,1,1,2) \text { and } \mu_{3}(1,1,2,2)
$$

A closed form expression for $p(m)$ is not known in general, and it has not been possible to obtain a general decomposition formula for the $n$-dimensional case.

For any $\boldsymbol{n}$ it is, in principle, possible to apply the theorem repeatedly and write down the solutions to the differential equations leading to a decomposition.

For example for $n=3$, if $s$ is the length of an edge, $A$ the area of a face and $V$ the volume of $S_{3}$, then

$$
3 \frac{d s}{s}=\frac{3}{2} \frac{d A}{A}=\frac{d V}{V}
$$

and by applying the theorem repeatedly as in II we obtain seven differential equations in the $\mu_{3}$ 's which on solution for functions varying as $V^{h}$ give:

$\mu_{3}=\frac{4}{3(h+3)(h+4)(3 h+10)(3 h+11)}\left\{\begin{array}{l}170 \mu_{3}(1,1,1,2)+178 \mu_{3}(1,1,2,2) \\ +606 \mu_{3}(1,1,2,3)+36 \mu_{3}(1,2,3,4)\end{array}\right\}$.

When we consider the volume of the convex hull of the four points we can show

$$
\mu_{3}(1,1,1,2)=\frac{3}{64} \quad \text { and } \mu_{3}(1,2,3,4)=\frac{1}{27}
$$

but the other two basic expectations have not been evaluated. They are the expected value of the volume of the tetrahedron formed by four points when there is:

(a) one point at random on each of two opposite edges of $S_{3}$, and two points at random in the interior of $S_{3}$

(b) one point at random in an edge of $S_{3}$, and one point at random in each of the two faces not incident with that edge, and the fourth point at random in the interior of $S_{3}$.

For higher values of $n$ an algorithm could easily be developed to obtain the decomposition into the linear combination of the $p(n+1)-1$ basic expectations. However in dimensions higher than two it seems that the basic expectations are difficult to evaluate.

Similarly for the $n$-dimensional parallelotope $\boldsymbol{P}_{\boldsymbol{n}}$ a decomposition could be achieved by repeated iteration of the theorem. We give some numerical values for $V_{\Delta_{n}}^{h}$. Firstly we give exact values of $V_{\Delta_{n}}^{2}$ calculated from the formula in II. 


\begin{tabular}{llllll}
\hline$n$ & 1 & 2 & 3 & 4 & 5 \\
\hline$V_{\Delta_{n}}^{2}$ & $1 / 6$ & $1 / 72$ & $3 / 4000$ & $8 / 270,000$ & $9.189 \times 10^{-7}$ \\
\hline
\end{tabular}

\begin{tabular}{llllll}
\hline$n$ & 6 & 7 & 8 & 9 & 10 \\
\hline$V_{\Delta_{n}}^{2}$ & $2.334 \times 10^{-8}$ & $5.027 \times 10^{-10}$ & $9.374 \times 10^{-12}$ & $1.539 \times 10^{-13}$ & $2.260 \times 10^{-15}$ \\
\hline
\end{tabular}

For $n=2$ the first four moments $V_{\Delta_{2}}^{h}$ of the distribution of $D\left(\Delta_{2}\right)$ are calculated from the formula in III.

\begin{tabular}{ccccc}
\hline$h$ & 1 & 2 & 3 & 4 \\
\hline$V_{\Delta_{2}}^{h}$ & $\frac{1}{12}$ & $\frac{1}{72}$ & $\frac{3}{1375}$ & $\frac{1}{9000}$ \\
\hline
\end{tabular}

For $n=3, \ldots, 10$ we give estimates of the first four moments $V_{\Delta_{n}}^{h}$ of the distribution of $D\left(\Delta_{n}\right)$ based on Monte-Carlo studies with samples of size 10,000 .

\begin{tabular}{rcccc}
\hline$n$ & $V_{\Delta_{n}}^{1}$ & $V_{\Delta_{n}}^{2}$ & $V_{\Delta_{n}}^{3}$ & $V_{\Delta_{n}}^{4}$ \\
\hline 3 & $1.763 \times 10^{-2}$ & $7.747 \times 10^{-4}$ & $5.971 \times 10^{-5}$ & $6.559 \times 10^{-6}$ \\
4 & $3.124 \times 10^{-3}$ & $2.712 \times 10^{-5}$ & $4.345 \times 10^{-7}$ & $1.023 \times 10^{-8}$ \\
5 & $5.185 \times 10^{-4}$ & $9.029 \times 10^{-7}$ & $3.960 \times 10^{-9}$ & $3.364 \times 10^{-11}$ \\
6 & $7.991 \times 10^{-5}$ & $2.440 \times 10^{-8}$ & $2.085 \times 10^{-11}$ & $3.699 \times 10^{-14}$ \\
7 & $1.109 \times 10^{-5}$ & $5.125 \times 10^{-10}$ & $5.993 \times 10^{-14}$ & $1.185 \times 10^{-17}$ \\
8 & $1.437 \times 10^{-6}$ & $1.093 \times 10^{-11}$ & $3.927 \times 10^{-16}$ & $3.324 \times 10^{-20}$ \\
9 & $1.780 \times 10^{-7}$ & $1.398 \times 10^{-13}$ & $2.631 \times 10^{-19}$ & $7.923 \times 10^{-25}$ \\
10 & $2.035 \times 10^{-8}$ & $1.954 \times 10^{-15}$ & $4.731 \times 10^{-22}$ & $1.863 \times 10^{-28}$ \\
\hline
\end{tabular}

The first four moments for the parallelogram $P_{2}$ are calculated from the formula in II

\begin{tabular}{ccccc}
\hline$h$ & 1 & 2 & 3 & 4 \\
\hline$V_{P_{2}}^{h}$ & $\frac{11}{144}$ & $\frac{53}{4800}$ & $\frac{149}{72,000}$ & $\frac{9}{19,600}$ \\
\hline
\end{tabular}




\section{REFERENCES}

1. M. W. Crofton, Probability, Encyclopaedia Britannica, ninth edition, (1885), 19768-788. 2. On the theory of local probability applied to straight lines drawn at random in a plane; etc. Philos. Trans. Roy. Soc. London, 158 (1868), 181-199.

3. M. G. Kendall, and P. A. P. Moran, Geometric Probability, Griffin and Co., London, 1963.

4. V. Klee, What is the expected volume of a simplex whose vertices are chosen at random from a given convex body? Amer. Math. Monthly 76 (1969), 286-288.

5. J. F. C. Kingman, Random secants of a convex body, J. Appl. Prob. 6 (1969), 660-672.

6. H. Ruben, and W. J. Reed, A more general form of a theorem of Crofton, J. Appl. Prob., 10, 479-482 (1973).

7. J. J. Sylvester, On a special class of questions on the theory of probabilities, British Assoc. Report, (1865), 8-9.

8. __ Question No. 1229, Educational Times, 18 (1865), p. 211.

9. __ Question No. 2531, Educational Times, 20 (1867) p. 208.

10. S. S. Wilks, Mathematical Statistics, Wiley 1962. Chapter 7.7, 177-182.

Received December 6, 1972. This work was in large part carried out at McGill University as part of the research for an M.Sc. thesis.

University OF BRITISH COLUMBIA 


\section{PACIFIC JOURNAL OF MATHEMATICS}

\section{EDITORS}

RICHARD ARENS (Managing Editor)

University of California

Los Angeles, California, 90024

\section{R. A. BEAUMONT}

University of Washington

Seattle, Washington 98105

\section{J. DugunDJI}

Department of Mathematics University of Southern California Los Angeles, California 90007

D. Gillbarg AND J. MilgRAM

Stanford University Stanford, California 94305

\section{ASSOCIATE EDITORS}

E. F. BECKENBACH

B. H. NEUMANN

SUPPORTING

UNIVERSITY OF BRITISH COLUMBIA

UNIVERSITY OF CALIFORNIA

MONTANA STATE UNIVERSITY

UNIVERSITY OF NEVADA

NEW MEXICO STATE UNIVERSITY

OREGON STATE UNIVERSITY

UNIVERSITY OF OREGON OSAKA UNIVERSITY
F. WOLF

K. YosHIDA

INSTITUTIONS

UNIVERSITY OF SOUTHERN CALIFORNIA

STANFORD UNIVERSITY

UNIVERSITY OF TOKYO

UNIVERSITY OF UTAH

WASHINGTON STATE UNIVERSITY

UNIVERSITY OF WASHINGTON

AMERICAN MATHEMATICAL SOCIETY

NAVAL WEAPONS CENTER

The Supporting Institutions listed above contribute to the cost of publication of this Journal, but they are not owners or publishers and have no responsibility for its content or policies.

Mathematical papers intended for publication in the Pacific Journal of Mathematics should be in typed form or offset-reproduced, (not dittoed), double spaced with large margins. Underline Greek letters in red, German in green, and script in blue. The first paragraph or two must be capable of being used separately as a synopsis of the entire paper. Items of the bibliography should not be cited there unless absolutely necessary, in which case they must be identified by author and Journal, rather than by item number. Manuscripts, in duplicate if possible, may be sent to any one of the five editors. Please classify according to the scheme of Math. Rev. Index to Vol. ${ }^{39}$. All other communications to the editors should be addressed to the managing editor, or Elaine Barth, University of California, Los Angeles, California, 90024.

100 reprints are provided free for each article, only if page charges have been substantially paid. Additional copies may be obtained at cost in multiples of 50 .

The Pacific Journal of Mathematics is issued monthly as of January 1966. Regular subscription rate: $\$ 72.00$ a year (6 Vols., 12 issues). Special rate: $\$ 36.00$ a year to individual members of supporting institutions.

Subscriptions, orders for back numbers, and changes of address should be sent to Pacific Journal of Mathematics, 103 Highland Boulevard, Berkeley, California 90708.

\section{PUBLISHED BY PACIFIC JOURNAL OF MATHEMATICS, A NON-PROFIT CORPORATION}

Copyright (C) 1974 by Pacific Journal of Mathematics

Manufactured and first issued in the U.S.A. 


\section{Pacific Journal of Mathematics}

\section{Vol. 54, No. $2 \quad$ June, 1974}

John Edward Coury, Walsh series with coefficients tending monotonically to

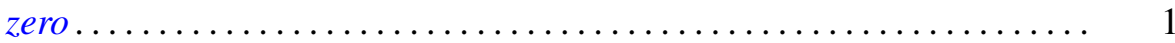

Patrick Michael Fitzpatrick and Walter Volodymyr Petryshyn, Fixed point theorems for multivalued noncompact acyclic mappings ............

Irving Leonard Glicksberg, More on Phragmén-Lindelöf for function

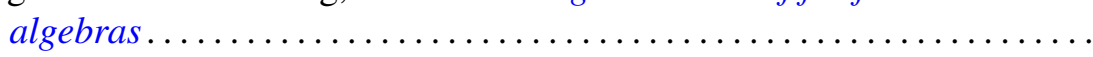

Adilson Goncalves, Structural constants. II .................. 39

Richard P. Gosselin, Closure theorems for affine transformation groups .... 53

Ralph Peter Grimaldi, Baer and UT-modules over domains ........... 59

Edward Grossman, On the prime ideal divisors of $\left(a^{n}-b^{n}\right) \ldots \ldots \ldots \ldots . \ldots 73$

A. Hedayat and Ester Seiden, On the theory and application of sum composition of Latin squares and orthogonal Latin squares.......... .

Gerald L. Itzkowitz, Continuous measures, Baire category, and uniform continuity in topological groups ......................... 115

Francis Masat, Right simple congruences on a semigroup ............ 127

Robert Harvey Oehmke, Right congruences and semisimplicity for Rees matrix semigroups..................................

Qazi Ibadur Rahman and Jan Stankiewicz, Differential inequalities and local valency . . . . . . . . . . . . . . . . . . . . . . . . . . . . . . . . . . . . . 165

William John Reed, Random points in a simplex ................ 183

Mohan S. Shrikhande, Strongly regular graphs and group divisible

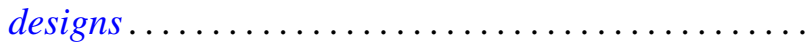

Zahava Shmuely, The structure of Galois connections ... . .

Robert C. Shock, Dual generalizations of the Artinian and Noetherian

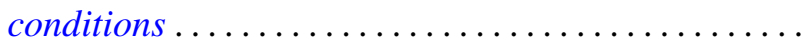

Arne Stray, Approximation and interpolation for some spaces of analytic functions in the unit disc ........................

Eldon Jon Vought, Monotone decompositions into trees of Hausdorff continua irreducible about a finite subset ............

James Wirth, The mapping cylinder axiom for WCHP fibrations ... 263

Gordon S. Woodward, Invariant means and ergodic sets in Fourier analysis... 